\title{
Robustness of Model Reference Adaptive Control Systems with Magnified Leakage Term
}

\author{
A-Cheng $W^{1}$, Li-Chen $F^{1,2}$ and Chen-Fa Hsu ${ }^{1}$ \\ ${ }^{1}$ Department of Electrical Engineering \\ ${ }^{2}$ Department of Computer Science and Information Engineering \\ National Taiwan University \\ Taipei, Tajwan, Republic of China
}

\begin{abstract}
This paper investigates the robustness of model reference adaptive control(MRAC) of a class of uncertain dynamical system using state information. The controller structure used here is quite standard in the literature of adaptive control, and the parameter update law adopted here incorporates some magnified leakage term like the well-known $\sigma$-modification[11]. But unlike MRAC of systems with only $1 / 0$ measurement, the scheme presented here can be robust to arbitrarily high level of matched uncertainties(besides its robustness to some degree of mismatched uncertainties). It is also worthwhile to point out that such scheme differs from those proposed in the literature of (adaptive) robust control is that the strong robustness is obtained under no high-gain condition and hence there is no phenomenon for the possibility of saturating the input channel. Furthermore, the present adaptive controller remains to have asymptotic tracking capability in the ideal case, which is hardly obtained by conventional (adaptive) robust controller under practical design.
\end{abstract}

\section{Introduction}

The control of uncertain systems has attracted the attention of system theorists for a long time. By this we mean that the system or, to be more precise, the model of the system, contains uncertain elements which may be nonlinear and/or time-varying and are unknown or imperfectly known. Furthermore, the control input may be contaminated by measurement error. Under such a circumstance, one tries to design a control such that the plant has certain satisfactory properties.

Recently, robustness of uncertain systems with matched uncertainties were proved by Chen and Leitmann [2], where the matched uncertainty with arbitrarily large strength is shown stabilizable by a fixed robust control law. They also showed that in the absence of matching assumptions the proposed control law remains a stabilizing one provided the uncertainty is small enough. While in the adaptive literature, Coreless and Leitmann [4] first construct a class of saturation-type adaptive robust controllers with state measurement error. The control gain is tuned adaptively to deal with the unknown uncertainty bound. In Chen [1] and Wu et al. [8], adaptive control algorithms utilizing concave function are presented to overcome the unknown uncertainty bound. In the absence of input uncertainty, two new classes of adaptive robust control schemes were developed by Chen [3]. Both uncertain dynamics and measurement noise are considered but at the price that the tracking error(state) $x(t)$ does not converge to the origin even in the absence of those non-idealities.

In this paper, we present a model reference adaptive control (MRAC) scheme which is robust to different types of uncertainties. The proposed adaptive controller adopted the standard structure [6] with a parameter update law augmented by some magnified leakage term similar to the well-known $\sigma$-modification [11]. This kind of modified update law is not new to the literature of MRAC with only $\mathrm{I} / \mathrm{O}$ information, i.e., state is not accessible. Therefore, the present scheme is shown to similarly possess the robustness to both matched and mismatched system uncertainties, which can be nonlinear and time-varying unlike [7], and the capability of asymptotic convergence when in the ideal case. Furthermore, the residual set of the tracking error(state) is such that the magnitude of the limiting time-average of the squared error is of the order of the levels of uncertainties and some design constant $\sigma_{0}$ inside the leakage term. But, here what is better for the present scheme is its robustness to arbitrarily high level of matched uncertainties, which is hardly obtained in the literature of MRAC[6][11]. It is, however, worthwhile to point out that the proceeding feature is obtained under no high-gain condition like what has usually been assumed explicitly or implicitly[1][3][8]. As a result, the control force does not have any indication to be large for possible saturation problem.

The layout of this paper is as follows : The first section is the introduction. The problem of the adaptive tracking control of the uncertain plants is formulated in section 2. The design and analysis of robust model reference adaptive control system is presented in section 3 . Section 4 is the simulation. Section 5 is the conclusion.

\section{Problem Formulation}

Let the class of plants to be controlled here be described as follows :

$$
\dot{x}=A_{0}(x, t) x+B_{0}(x, t) u, \quad x\left(t_{0}\right)=x_{0}
$$


where $x \in R^{n}$ is the state vector, $u \in R^{m}$ is the vector of control inputs, $A_{0}(\cdot, \cdot): R^{n} \times R_{+} \rightarrow R^{n \times n}$ and $B(\cdot, \cdot): R^{n} \times R_{+} \rightarrow R^{n \times m}$ are $C^{\mathbf{l}}$ (continuously differentiable) with respect to both $x$ and $t$, and for any control $u=u(x, t)$ which is also $C^{1}$ with respect to both $x$ and $t$ the solution trajectory exists. Now suppose the control task is to drive the system (2.1) to follow the following reference model :

$$
\dot{x}_{m}=A_{m} x_{m}+B_{m} r, \quad x_{m}\left(t_{0}\right)=x_{m_{0}}
$$

where $x_{m} \in R^{n}$ denotes the reference state, $r \in R^{m}$ denotes the vector of reference exogenous inputs, and $\left(A_{m}, B_{m}\right)$ is a stabilizable pair such that $A_{m}$ itself is a Hurwitz matrix. Then, the problem to be solved here is clearly how to design the vector of plant inputs $u$ such that the control task can be fulfilled, namely,

$$
x(t) \rightarrow x_{m}(t) \quad \text { as closely as possible when } \quad t \rightarrow \infty
$$

This problem is generally hard to solve and can be made more tractable if the following assumptions about (2.1) are satisfied:

(A1) The system (2.1) can be rearranged in the following form :

$$
\begin{aligned}
\dot{x}= & \left(A_{m}+B_{m} \theta^{*}\right) x+\mu_{1} \Delta A_{1}(x, t) \\
& +\left(B_{m}+\mu_{1} \Delta B_{1}(x, t)\right) u+\mu_{2} B_{m} \Delta A_{2}(x, t),
\end{aligned}
$$

where $\mu_{1}, \mu_{2} \in R_{+}, \mu_{1} \Delta A_{1}(x, t)$ and $\mu_{1} \Delta B_{1}(x, t) u$ are generally termed as mismatched uncertainties whereas $\mu_{2} B_{m} \Delta$ $A_{2}(x, t)$ is generally called matched uncertainties, and $\theta^{*} \in$ $R^{m \times n}$ is not known a priori so that $B_{\mathrm{m}} \theta^{*} x$ represents the parametric uncertainty (in contrast with the former nonparametric uncertainties with levels $\mu_{1}$ and $\mu_{2}$, respectiveIy).

(A2) The upper bound on $\left\|\theta^{*}\right\|_{1}$ is assumed to be known a priori as $\theta_{\max }\left(\|\cdot\|_{1}\right.$ denotes the one-norm of the argument vector or the induced one-norm of the argument matrix).

(A3) The nonlinear time-varying uncertainties in (2.3) satisfy the following cone-bounded conditions :

$$
\begin{aligned}
& \sup _{t \geq 0}\left\|\Delta A_{1}(x, t)\right\| \leq g_{1}\|x\|+g_{2} ; \\
& \sup _{t \geq 0}\left\|\Delta A_{2}(x, t)\right\| \leq g_{3}\|x\|+g_{4} ; \\
& \sup _{t \geq 0}\|\Delta B(x, t)\| \leq g_{5},
\end{aligned}
$$

for some $g_{i} \geq 0, i=1,2, \cdots, 5$, where $\|\cdot\|$ denotes either the matrix norm or the Euclidean vector norm depending on its argument.

This new form (2.3) with the system properties (2.4) implies that the system behavior of the original plant in fact is dominated by that of a linear plant :

$$
\dot{x}=\left(A_{m}+B_{m} \theta^{*}\right) x+B_{m} u
$$

provided $\mu_{1}$ and $\mu_{2}$ are small, or equivalently that (2.5) is the nominal model of the original plant (2.1).

\section{Remarks :}

(1) It should not be difficult to rewrite (2.1) into the form (2.3). In general, we can first linearize (2.1) around the origin to obtain

$$
\dot{x}=A x+B u
$$

where $A=\left.\frac{\partial A_{0}(x, t)}{\partial x}\right|_{x=0, t=0}$ and $B=\left.\frac{\partial B_{0}(x, t)}{\partial x}\right|_{x=0, t=0}$. Suppose that $(A, B)$ is a controllable pair, then there exists a matrix $K \in R^{m \times n}$ such that $A-B K=A_{m}$ is a Hurwitz matrix. If we further define $B_{m}=B$, then the linearized system is indeed (2.5) with $\theta^{*}=K$.

In the second, let $A_{0}(x, t) x-A x=\mu_{1} \Delta A_{1}(x, t)+\mu_{2} B_{m} \Delta A_{2}$ $(x, t)$ and $B_{0}(x, t)-B=\mu_{1} \Delta B_{1}(x, t)$, we readily obtain (2.3). It is noteworthy that $\Delta A_{1}(x, t), \Delta A_{2}(x, t)$, and $\Delta B_{1}$ $(x, t)$ can be arbitarily fast time-varying with the inequalities (2.4) being satisfied.

(2) One should note that $B_{m} \theta^{*} x$ is actually one kind of matched uncertainty. Therefore, the expressions (2.1), (2.3), (2.5) implicitly imply that $\left(A_{m}+B_{m} \theta^{*}\right) x$ represents the maximum parameterizable information available from $A_{0}(x, t)$ so that $\mu_{2}$ (level of matched uncertainty) can normally be kept small.

Based on the expression (2.3) of the plant, we can derive the error system conforming the control task by subtracting (2.2) from (2.3), namely,

$$
\begin{aligned}
\dot{e}= & A_{m} e+B_{m}\left(u+\theta^{*} x-r+\mu_{2} \Delta A_{2}(x, t)\right) \\
& +\mu_{1}\left(\Delta A_{1}(x, t)+\Delta B_{1}(x, t) u\right)
\end{aligned}
$$

where $e \triangleq x-x_{m}$ will be referred to as error state. Now, we can restate the problem in the context of robust model reference adaptive control as follows : devise a continuous control law $u=u(\hat{\theta}, x, t)$, where $\hat{\theta}$ is an estimate of $\theta^{*}$, as well as a parameter update law $\dot{\hat{\theta}}=\Phi(\hat{\theta}, x, t)$ so that $e(t)$ approaches zero asymptotically in the ideal case, i.e., $\mu_{1}=\mu_{2}=0$, and $e(t)$ converges to a small residue set asymptotically when $\mu_{1}$ and $\mu_{2}$ are not zero but generally small, i.e., the control has to be robust to the existence of nonparametric uncertainties. Furthermore, the limiting time average of the squared error satisfies

$$
\lim _{T \rightarrow \infty} \sup _{T \geq 0} \frac{1}{T} \int_{t_{0}}^{t_{0}+T}\|e(\tau)\|^{2} d \tau \leq K\left(\mu_{1}, \mu_{2}, \sigma_{0}\right)
$$

where $K$ is an increasing function with $\mu_{1}, \mu_{2}$, and $\sigma_{0}$ separately, and $K(0,0,0)=0$. 


\section{Robust Model Reference Adaptive Control}

Under the problem formulation in the previous section, it is quite natural to devise the control law as :

$$
u(\hat{\theta}, x, t)=-\hat{\theta} x+r
$$

using certainty equivalence principle [6]. By this design, we emphasize that this is really a continuous control so long as the parameter update law is well specified and the reference inputs are themselves continuous.

Now, let the parameter update law be chosen as :

$$
\dot{\phi}=\frac{d}{d t}\left(\hat{\theta}-\theta^{*}\right)=\Phi(\hat{\theta}, x, t)=-B_{m}^{T} P e x^{T}-\hat{\theta} \sigma
$$

where $P \in R^{n \times n}$ is a positive definite matrix satisfying the Lyapunov equation $\frac{1}{2}\left(A_{m}^{T} P+P A_{m}\right)=-Q$ for some positive definite niatrix $Q$, and $\sigma=\operatorname{diag}\left(\sigma_{1}, \cdots, \sigma_{n}\right)$ with

$$
\sigma_{i}=\left\{\begin{array}{ll}
0 & \text { if }\left\|\hat{\theta}_{i}\right\|_{1} \leq n \theta_{\max }, i=1,2, \cdots, n \\
\sigma_{0}(1+\|e\|\|x\|) & \text { otherwise }
\end{array},\right.
$$

where $\hat{\theta}_{i}$ denotes the i-th column of $\hat{\theta}$.

To investigate the effectiveness of this adaptive control, we adopt the popular Lyapunov analysis, i.e., by constructing a Lyapunov function $V(e, \phi)$ as follows :

$$
V(e, \phi)=\frac{1}{2}\left(e^{T} P e+\operatorname{Tr}\left(\phi^{T} \phi\right)\right)
$$

and then evaluating its time derivative along the solution trajectories of (2.6), (3.2). The investigation will first be performed in the ideal case in which the error system (2.6) will become

$$
\dot{e}=A_{m} e-B_{m} \phi x
$$

so that the time derivative of (3.4) becomes:

$$
\begin{aligned}
\dot{V}(e, \phi)= & {\left[\frac{1}{2} e^{T}\left(A_{m}^{T} P+P A_{m}\right) e-e^{T} P B_{m} \phi x\right] } \\
& +\operatorname{Tr}\left[\phi^{T}\left(-B_{m}^{T} P e x^{T}-\hat{\theta} \sigma\right)\right] \\
= & -e^{T} Q e-\operatorname{Tr}\left[\phi^{T} \hat{\theta} \sigma\right] \\
\leq & -q\|e\|^{2}-\sum_{i=1}^{n} \sigma_{i}\left(\left\|\hat{\theta}_{i}\right\|^{2}-\left\|\hat{\theta}_{i}\right\|_{1}\left\|\theta_{i}^{*}\right\|_{1}\right),
\end{aligned}
$$

where $q=\lambda_{\min }(Q)$ and $\theta_{i}^{*}$ denotes the i-th column of $\theta^{*}$. It is clear from (3.3) that

$$
\sigma_{i}\left(\left\|\hat{\theta}_{i}\right\|^{2}-\left\|\hat{\theta}_{i}\right\|_{1}\left\|\theta_{i}^{*}\right\|_{1}\right) \geq \frac{\sigma_{i}}{n}\left\|\hat{\theta}_{i}\right\|_{1}\left(\left\|\hat{\theta}_{i}\right\|_{1}-n\left\|\theta_{i}^{*}\right\|_{1}\right)
$$

and, hence, (3.6) satisfies $\dot{V} \leq-q\|e\|^{2}$. Then, boundedness of all signals is clear, and the asymptotic convergence of the error state to the origin can be concluded via Barbalat's lemma [6].

But in the presence of nonparametric uncertainties, (2.6) becomes

$$
\begin{aligned}
\dot{e}= & A_{m} e-B_{m} \phi x+\mu_{2} B_{m} \Delta A_{2}(x, t) \\
& +\mu_{1}\left(\Delta A_{1}(x, t)+\Delta B_{1}(x, t) u\right),
\end{aligned}
$$

and the investigation will proceed with a different Lyapunov function

$$
V(e, \phi)=\frac{1}{2}\left(e^{T} P e+\operatorname{Tr}\left\{(\phi+\pi \zeta)^{T}(\phi+\pi \zeta)\right\}\right)
$$

where $\zeta=B_{m}^{T} P$ and $\pi$ is a positive constant to be specified. To evaluate the time derivative of (3.9) along the solution trajectories of (3.8), instead of (3.5), we obtain

$$
\begin{aligned}
\dot{V}(e, \phi)= & -e^{T} Q e+\operatorname{Tr}\left\{(\pi \zeta)^{T}\left(-B_{m}^{T} P e x^{T}-\hat{\theta} \sigma\right)\right\} \\
& -\operatorname{Tr}\left[\phi^{T} \hat{\theta} \sigma\right]+e^{T} P\left[\mu_{1}\left(\Delta A_{1}+\Delta B_{1} u\right)+\mu_{2} B_{m} \Delta A_{2}\right] \\
= & -e^{T} Q e-\operatorname{Tr}\left\{(\phi+\pi \zeta)^{T} \hat{\theta} \sigma\right\} \\
& -\pi\left(\left\|e_{a}\right\|^{2}+e_{a}^{T} e_{a m}\right) \\
& +e^{T} P\left[\mu_{1}\left(\Delta A_{1}+\Delta B_{1} u\right)+\mu_{2} B_{m} \Delta A_{2}\right],
\end{aligned}
$$

where $e_{a} \triangleq B_{m}^{T} P e$ and $e_{a m}=B_{m}^{T} P x_{m}$. Now, suppose $T>0$ is such that $\forall t \in\left[t_{0}, t_{0}+T\right),\left\|\hat{\theta}_{i}(t)\right\|_{1} \leq n \theta_{\max }, \forall i=1, \cdots, n$. Then, (3.10) can be further rewritten as :

$$
\begin{aligned}
\dot{V}(e, \phi) \leq & -q\|e\|^{2}-\pi\left(\left\|e_{a}\right\|^{2}+e_{a}^{T} e_{a m}\right)+\mu_{1}\|e|\||| P\| \\
& \left\{\left(g_{1}\|x\|+g_{2}\right)+g_{5}\left(n \theta_{m a x}\|x\|_{1}+\|r\|\right)\right\} \\
& +\mu_{2}\left\|e_{a}\right\|\left(g_{3}\|x\|+g_{4}\right)
\end{aligned}
$$

where $q=\lambda_{\min }(Q)$, and we use the fact that $\|\hat{\theta} x\| \leq\left(\sum_{i}\left|x_{i}\right|\right)$ $\max _{j}\left\|\hat{\theta}_{j}\right\|_{1} \leq n \theta_{\text {max }}\|x\|_{1}$. By some mathematical manipulation$\mathrm{s}$, the above equation can be reexpressed as :

$$
\begin{aligned}
\dot{V}(e, \phi) \leq & -q\|e\|^{2}-\pi\left\|e_{a}\right\|^{2}+\mu_{2} g_{3}\left\|e_{a}\right\|\|e\|+\pi e_{a}^{T} e_{a m} \\
& +\mu_{2} g_{3}\left\|e_{a}\right\|\left\|x_{m}\right\|+\mu_{2} g_{4}\left\|e_{a}\right\|+\mu_{1}\|e\|\|\| P \|\left\{g_{1}\|e\|\right. \\
& +\left(g_{1}\left\|x_{m}\right\|+g_{2}\right)+g_{5}\left(n^{\frac{3}{2}} \theta_{\text {max }}\left\|e_{\sharp}\right\|\right. \\
& \left.\left.+\|r\|+n^{\frac{3}{2}} \theta_{\max }\left\|x_{m}\right\|\right)\right\} .
\end{aligned}
$$

Now, we complete the squares of the terms involving $e_{a}$ to obtain

$$
\begin{aligned}
\dot{V}(e, \phi) \leq & -\left(q-\mu_{1}\left(g_{1}\|P\|+g_{5} n^{\frac{3}{2}} \theta_{\max }\right)-\frac{\mu_{2}^{2} g_{3}^{2}}{4 \pi}\right)\|e\|^{2} \\
& -\pi\left(\left\|e_{a}\right\|-\frac{\mu_{2} g_{3}}{2 \pi}\|e\|\right)^{2}+\left\|e_{a}\right\|\left(\pi\left\|e_{a m}\right\|\right. \\
& \left.+\mu_{2} g_{3}\left\|x_{m}\right\|+\mu_{2} g_{4}\right)+\mu_{1}\|e\|\|P\| \\
& \left\{\left(g_{1}+n^{\frac{3}{2}} \theta_{\max }\right)\left\|x_{m}\right\|+g_{2}+g_{5}\|r\|\right\}
\end{aligned}
$$

Because $\pi$ is a fictitious constant, i.e., for analysis only, we can choose it appropriately large so that $\frac{\mu_{2}^{2} g_{3}^{2}}{4 \pi}<\frac{1}{3} q$. Since $g_{1}, g_{5},\|P\|, n$, and $\theta_{\max }$ are constants, there exists $\bar{\mu}_{1}$ such that, for all $\mu_{1} \in$ $\left[0, \bar{\mu}_{1}\right), \mu_{1}\left(g_{1}\|P\|+g_{5} n^{\frac{3}{2}} \theta_{\max }\right)<\frac{1}{3} q$, then we can let $q_{1}=q-$ $\mu_{1}\left(g_{1}\|P\|+g_{5} n^{\frac{3}{2}} \theta_{\text {max }}\right)-\frac{\mu_{2}^{2} g_{3}^{2}}{4 \pi}>\frac{1}{3} q>0$ so that (3.13) can be reduced to

$$
\dot{V}(e, \phi) \leq-q_{1}\|e\|^{2}+k_{1}\left(\pi, \mu_{1}, \mu_{2}, \theta_{\max }, t\right)\|e\|,
$$

where $k_{1}(\cdot)=\left\|B^{T} P\right\|\left(\pi\left\|e_{a m}\right\|+\mu_{2}\left(g_{3}\left\|x_{m}\right\|+\mu_{2} g_{4}\right)\right)+\mu_{1}\|P\|\left\{\left(g_{1}+\right.\right.$ $\left.\left.n^{\frac{3}{2}} \theta_{\max }\right)\left\|x_{m}\right\|+g_{2}+g_{5}\|r\|\right\}$ is a bounded function with respec$t$ to its arguments. Then, subtracting from and adding to the right hand side of (3.14) $\sigma_{0} \operatorname{Tr}\left\{(\phi+\pi \zeta)^{T}(\phi+\pi \zeta)\right\}$ and using the 
inequality $\operatorname{Tr}\left\{(\phi+\pi \zeta)^{T}(\phi+\pi \zeta)\right\} \leq 4 n \theta_{\max }^{2}+2 \pi(n+1) \theta_{\max } \sum_{i}$ $\left\|\zeta_{i}\right\|+\pi^{2} \sum_{i}\left\|\zeta_{i}\right\|^{2}$, we obtain

$$
\begin{aligned}
\dot{V}(e, \phi) \leq & -q_{1}\|e\|^{2}-\sigma_{0} \operatorname{Tr}\left\{(\phi+\pi \zeta)^{T}(\phi+\pi \zeta)\right\} \\
& +K_{1}\|e\|+4 n \sigma_{0} \theta_{\max }^{2}+2 \sigma_{0} \pi(n+1) \theta_{\max } \sum_{i}\left\|\zeta_{i}\right\| \\
& +\sigma_{0} \pi^{2} \sum_{i}\left\|\zeta_{i}\right\|^{2}
\end{aligned}
$$

where $K_{1}=\sup _{t \geq 0} k_{1}\left(\pi, \mu_{1}, \mu_{2}, \theta_{\max }, t\right)$ and $\zeta_{i}$ represent the $i$-th column of $\zeta$. From (3.9), there exist $\gamma_{1}, \gamma_{2}>0$ such that

$$
\dot{V} \leq-\gamma_{1} V+\gamma_{2} V^{\frac{1}{2}}+\eta_{1},
$$

where

$$
\begin{aligned}
\gamma_{1}= & 2 \min \left\{q_{1} \lambda_{\max }^{-1}(P), \sigma_{0}\right\} \\
\gamma_{2}= & K_{1}\left(\frac{1}{2} \lambda_{\min }(P)\right)^{-\frac{1}{2}} \\
\eta_{1}= & 4 n \sigma_{0} \theta_{\max }^{2}+2 \sigma_{0} \pi(n+1) \theta_{\max } \sum_{i}\left\|\zeta_{i}\right\| \\
& +\sigma_{0} \pi^{2} \sum_{i}\left\|\zeta_{i}\right\|^{2}
\end{aligned}
$$

Certainly, $V$ must eventually fall into a residual interval $\left[0, R_{1}\right]$, where $R_{1}=\left(\frac{\gamma_{1}+\sqrt{\gamma_{2}^{2}+4 \gamma_{1} \eta_{1}}}{2 \gamma_{1}}\right)^{2}$, provided $T=\infty$. Next, we show that the similar inequality will hold also when $\left\|\hat{\theta}_{i}(t)\right\|_{1}>n \theta_{\max }$ for all $i \in N_{1} \subset\{1, \cdots, n\}=N$ and $t>t_{0}+T$.

In this case, (3.10) can be replaced by

$$
\begin{aligned}
\dot{V}(e, \phi) \leq & -q\|e\|^{2}-\pi\left(\left\|e_{a}\right\|^{2}+e_{a}^{T} e_{a m}\right)+\mu_{1}\|e\|\|P\| \\
& \left\{\left(g_{1}\|x\|+g_{2}\right)+g_{5}\left(n^{\frac{1}{2}}\|\hat{\theta}\|\left\|_{1}\right\| x\|+\| r \|\right)\right\} \\
& +\mu_{2}\left\|e_{a}\right\|\left(g_{3}\|x\|+g_{4}\right) \\
& -\operatorname{Tr}\left\{(\phi+\pi \zeta)^{T} \hat{\theta} \sigma\right\}
\end{aligned}
$$

Likewise, we complete the square of the terms involving $e_{a}$ to obtain

$$
\begin{aligned}
\dot{V}(e, \phi) \leq & -\left(q-\mu_{1} g_{1}\|P\|-\frac{\mu_{2}^{2} g_{3}^{2}}{4 \pi}\right)\|e\|^{2} \\
& +\mu_{1}\|e\|\|P\|\left(g_{1}\left\|x_{m}\right\|+g_{2}+g_{5}\|r\|\right) \\
& +\pi\left\|B^{T} P\right\|\left[\mu_{2}\left(g_{3}\left\|x_{m}\right\|+g_{4}\right)+\left\|e_{a m}\right\|\right]\|e\| \\
& +\mu_{1} g_{5} n^{\frac{1}{2}}\|e\|\|P\|\|\hat{\theta}\|_{1}\|x\| \\
& -\sigma_{0}(1+\|e\|\|x\|) \sum_{i \in N_{1}}\left(\phi_{i}+\pi \zeta_{i}\right)^{T} \hat{\theta}_{i}
\end{aligned}
$$

where $\phi_{i}$ denotes the $\mathrm{i}$-th column of $\phi$. For proceeding further, we use the fact that

$$
\begin{gathered}
-\sigma_{0}(1+\|e\|\|x\|) \sum_{i \in N_{1}}\left(\phi_{i}+\pi \zeta_{i}\right)^{T} \hat{\theta}_{i} \leq-\frac{1}{2} \sigma_{0} \sum_{i \in N_{1}}\left\|\phi_{i}+\pi \zeta_{i}\right\|^{2} \\
+\frac{1}{2} \sigma_{0} \sum_{i \in N_{1}}\left\|\theta_{i}^{*}-\pi \zeta_{i}\right\|^{2}-\frac{1}{2} \sigma_{0}\|e\|\|x\|\|\hat{\theta}\|_{1}^{2} \\
+\frac{1}{2} \sigma_{0}\|e\|\|x\| \sum_{i \in N_{1}}\left\|\theta_{i}^{*}-\pi \zeta_{i}\right\|^{2}
\end{gathered}
$$

to derive the following

$$
\begin{aligned}
\dot{V}(e, \phi) \leq & -\left(q-\mu_{1} g_{1}\|P\|-\frac{\mu_{2}^{2} g_{3}^{2}}{4 \pi}-\frac{1}{2} \sigma_{0} \sum_{i \in N_{1}}\left\|\theta_{i}^{*}-\pi \zeta_{i}\right\|^{2}\right)\|e\|^{2} \\
& -\|e\|\|x\|\|\hat{\theta}\| \|_{1}\left(\frac{1}{2} \sigma_{0}\|\hat{\theta}\|_{1}-\mu_{1} g_{5} n^{\frac{1}{2}}\right) \\
& +k_{2}\left(\pi, \mu_{1}, \mu_{2}, \sigma_{0}, t\right)\|e\|-\frac{1}{2} \sigma_{0} \sum_{i \in N_{1}}\left\|\phi_{i}+\pi \zeta_{i}\right\|^{2} \\
& +\frac{1}{2} \sigma_{0} \sum_{i \in N_{1}}\left\|\theta_{i}^{*}-\pi \zeta_{i}\right\|^{2}
\end{aligned}
$$

where $k_{2}(\cdot)=\left\|B^{T} P\right\|\left(\mu_{2}\left(g_{3}\left\|x_{m}\right\|+g_{4}\right)+\pi\left\|e_{a m}\right\|\right)+\mu_{1}\|P\|\left(g_{1}\left\|x_{m}\right\|\right.$ $\left.+g_{5}\|r\|+g_{2}\right)+\frac{1}{2} \sigma_{0}\left\|x_{m}\right\| \sum_{i \in N_{1}}\left\|\theta_{i}^{*}-\pi \zeta_{i}\right\|^{2}$. Since $\left\|\hat{\theta}_{i}\right\|_{1}>$ $n \theta_{\max }$ for every $i \in N_{1}$, we known that $\|\hat{\theta}\|_{1}=\max _{i}\left\|\hat{\theta}_{i}\right\|_{1}$ $\geq n \theta_{\text {max }}$. Obviously, choosing $\bar{\mu}_{2}=\frac{\alpha_{0} n^{\frac{1}{2}} \theta_{\max }}{2 g_{\mathrm{s}}}$ can guarantee that $\frac{1}{2} \sigma_{0}\|\hat{\theta}\|_{1}-\mu_{1} g_{5} n^{\frac{1}{2}}>0$ for all $\mu_{1} \in\left[0, \bar{\mu}_{2}\right)$. If we define $q_{2}=q-\mu_{1} g_{1}\|P\|-\frac{\mu_{2}^{2} g_{3}^{2}}{4 \pi}-\frac{1}{2} \sigma_{0} \sum_{i \in N_{1}}\left\|\theta_{i}^{*}-\pi \zeta_{i}\right\|^{2}$, then certainly we can design $\pi$ and $\sigma_{0}$ to make $\frac{\mu_{2}^{2} g_{3}^{2}}{4 \pi}$ and $\frac{1}{2} \sigma_{0} \sum_{i \in N_{1}}\left\|\theta_{i}^{*}-\pi \zeta_{i}\right\|^{2}$ both less than $\frac{1}{4} q$. Furthermore, if we choose $\bar{\mu}_{3}=\frac{q}{4 g_{1}|| P \|}$, then for all $\mu_{1} \in\left[0, \bar{\mu}_{3}\right)$ we known that $\mu_{1} g_{1}\|P\|<\frac{1}{4} q$ and, hence, $q_{2}>\frac{1}{4} q>0$ for all $\mu_{1} \in\left[0, \min \left(\bar{\mu}_{2}, \bar{\mu}_{3}\right)\right)$ so that $(3.18)$ can be simplified as

$$
\begin{aligned}
\dot{V}(e, \phi) \leq & -q_{2}\|e\|^{2}+K_{2}\|e\|-\frac{1}{2} \sigma_{0} \sum_{i \in N_{1}}\left\|\phi_{i}+\pi \zeta_{i}\right\|^{2} \\
& +\frac{1}{2} \sigma_{0} \sum_{i \in N_{1}}\left\|\theta_{i}^{*}-\pi \zeta_{i}\right\|^{2} \\
\leq & -q_{2}\|e\|^{2}+K_{2}\|e\|-\frac{1}{2} \sigma_{0} \sum_{i}\left\|\phi_{i}+\pi \zeta_{i}\right\|^{2} \\
& +\frac{1}{2} \sigma_{0}\left(\sum_{i \in N_{1}}\left\|\theta_{i}^{*}-\pi \zeta_{i}\right\|^{2}\right. \\
& \left.+\sum_{i \in N_{2}}\left\|\phi_{i}+\pi \zeta_{i}\right\|^{2}\right),
\end{aligned}
$$

where $K_{2}=\sup _{t \geq 0} k_{2}\left(\pi, \mu_{1}, \mu_{2}, \sigma_{0}, t\right)$ and $N_{2}$ is the subset of $N$ such that $N_{1}$ and $N_{2}$ are disjointed and $N_{1} \cup N_{2}=N$. For convenience of presentation, we define

$$
\begin{aligned}
\gamma_{3} & =\min \left\{2 q_{2} \lambda_{\max }^{-1}(P), \sigma_{0}\right\} \\
\gamma_{4} & =K_{2}\left(\frac{1}{2} \lambda_{\min }(P)\right)^{-\frac{1}{2}} \\
\eta_{2} & =\frac{1}{2} \sigma_{0} \sum_{i \in N_{3}}\left\|\theta_{i}^{*}-\pi \zeta_{i}\right\|^{2}+\frac{1}{2} \eta_{1}
\end{aligned}
$$

to further reduce (3.20) to

$$
\dot{V}(e, \phi) \leq-\gamma_{3} V+\gamma_{4} V^{\frac{1}{2}}+\eta_{2}
$$

Now, we can conclude from (3.16) and (3.21) that the value of $V$ defined in (3.9) will eventually fall into a residual interval $\left[0, R_{0}\right]$, where $R_{0}=\max \left[R_{1}, R_{2}\right]$ with $R_{2}=\left(\frac{\gamma_{3}+\sqrt{\gamma_{1}^{2}+4 \gamma_{3} \eta_{2}}}{2 \gamma_{3}}\right)^{2}$, and hence that the global stability of the adaptive system is established. Now, we are in a position to present the main results of this paper in the following theorem:

Main Theorem : There exist $\mu_{1}^{*}>0$ such that for all $\mu_{1} \in$ $\left[0, \mu_{1}^{*}\right)$ and under the adaptive control law (3.1) (3.3), all signals in the closed-loop systems (2.6) remain globally uniformly bounded. In addition, the state tracking error will converge to 
the residual set

$$
\Omega_{e}=\left\{e: \lim _{T \rightarrow \infty} \sup _{T \geq 0} \frac{1}{T} \int_{t_{0}}^{t_{0}+T}\|e(\tau)\|^{2} d \tau \leq K_{3}\left(\mu_{1}, \mu_{2}, \sigma_{0}\right)\right\}
$$

where $K_{3}$ is a positive function of the order $\mu_{1}, \mu_{2}$, and $\sigma_{0}$, i.e., $K_{3}$ is an increasing function with $\mu_{1}, \mu_{2}$, and $\sigma_{0}$ separately, and $K_{3}(0,0,0)=0$. Moreover, in the absence of uncertainties, the state tracking error will converge to zero asymptotically.

Proof : Please refer to [10].

\section{Remarks :}

(1) The precondition for the global stability of the adaptive system is that $\mu_{1}$ must be small enough, which implies that the mismatched uncertainties have to be small. However, there is no constraint on the strength of the matched uncertainties. Therefore, the matched uncertainties can not destroy the global stability of the closed-loop system while the parameter update law is turned on. Nevertheless, the larger the matched uncertainties are, the larger $\mu_{2}$ and, hence, $\pi$ are and so that the tracking performance degrades because the size of the residual set grows.

(2) The magnified leakage term in adaptation law (3.2) pulls the drifted parameters back can generally avoid the problem of instability, which is very similar to the case of $\mathrm{M}$ RAC systems with only I/O measurements. Notwithstanding this, it may lead to conservative bounds associated with (3.20) and, perhaps, the adaptive system may have fluctuating transient. Since the bound obtained by Lyapunov function analysis is usually not as tight as the one the adaptive system actually has, we claim that the transient behavior of the proposed scheme is anticipated to be good as will be observed in the illustrative simulated examples in the next section.

\section{Simulation Examples}

To illustrate effectiveness of the scheme proposed in this paper, we consider the following system with some nonlinear uncertain dynamics :

$$
\begin{gathered}
{\left[\begin{array}{c}
\dot{x}_{1}(t) \\
\dot{x}_{2}(t)
\end{array}\right]=\left[\begin{array}{ll}
0 & 1 \\
1 & 0
\end{array}\right]\left[\begin{array}{l}
x_{1}(t) \\
x_{2}(t)
\end{array}\right]+\left[\begin{array}{c}
\mu_{1} \sin \left(x_{1}(t)\right) \\
\mu_{2}\left(x_{2} \cos \left(x_{1}(t)\right)+1\right)
\end{array}\right]} \\
+\left(\left[\begin{array}{ll}
1 & 0 \\
0 & 1
\end{array}\right]+\mu_{1}\left[\begin{array}{cc}
0 & 0 \\
0 & \cos \left(x_{2}(t)\right)
\end{array}\right]\right)\left[\begin{array}{l}
u_{1}(t) \\
u_{2}(t)
\end{array}\right]
\end{gathered}
$$

where $\mu_{1}=0.01$ and $\mu_{2}=10$. A simple reference model is chosen as :

$$
\left[\begin{array}{c}
\dot{x}_{m 1}(t) \\
\dot{x}_{m 2}(t)
\end{array}\right]=\left[\begin{array}{cc}
-1 & 1 \\
-1 & -2
\end{array}\right]\left[\begin{array}{l}
x_{m 1}(t) \\
x_{m 2}(t)
\end{array}\right]+\left[\begin{array}{ll}
1 & 0 \\
0 & 1
\end{array}\right]\left[\begin{array}{l}
r_{1} \\
r_{2}
\end{array}\right]
$$

with $r_{1}=10$, and $r_{2}=-10$. Simulation plots are depicted in Fig. 1 where satisfactory tracking performance is shown.

Another physical example in [3] for the control of pendulum motion is given as :

$$
\dot{x}(t)=A x(t)+B u(t)+(f-A x(t))
$$

with $x(t)=\left[x_{1}(t), x_{2}(t)\right]^{T}$, where

$$
A=\left[\begin{array}{cc}
0 & 1 \\
-2 & -3
\end{array}\right], B=\left[\begin{array}{l}
0 \\
1
\end{array}\right], f=\left[\begin{array}{c}
x_{2} \\
-\sin (x)-q(t) \cos (x)
\end{array}\right]
$$

and $q(t)=$ random $[-1,1]$. Since the controller in [3] is only for regulation, we choose the reference model to be

$$
\dot{x}_{m}(t)=A x_{m}(t)
$$

and the Lyapunov matrix $P$ to be

$$
P=\left[\begin{array}{ll}
\frac{5}{2} & \frac{1}{2} \\
\frac{1}{2} & \frac{1}{2}
\end{array}\right]
$$

The control force used in the scheme in [3](Fig.(2.d)) is apparently larger than the one used in the scheme proposed here (Fig.(2.c)) since the former is with high gain nature. In Fig.(2.a) and Fig.(2.b), the trajectories of the states resulting from the present scheme are depicted in solid line whereas those resulting from the scheme in [3] are in dashed line. Although applying the smaller control force, the present scheme in this paper seems to yield slightly faster transient response.

\section{Conclusion}

This paper presents an MRAC for a class of dynamical systems with nonlinear time-varying uncertainties. Under the assumption of state accessibility, the robustness against input, mismatched, and arbitrarily high level of matched uncertainties is shown. Further, the present control retains the capability of asymptotic convergence of the tracking error (state) in the ideal case. But in the presence of uncertainties, the residual tracking error is such that the limiting time-average of its square is of the order of the level of uncertainties and the design constant $\sigma_{0}$. Since no high gain concept is adopted in the presented scheme, the control force is generally more acceptable than those generated by the schemes in $[1][3][8]$, which is supported by the numerical simulation provided in the end. Ongoing research will concentrate on the development of the observer-based adaptive robust control scheme for a more practical environment for implementation.

\section{Reference}

[1] Chen, Y. H., "Modified Adaptive Robust Control System Design", Int. J. Control, Vol. 49, No. 6, pp. 1869-1882, 
1989.

[2] Chen, Y. H., "Robustness of Uncertain Systems in the Absence of Matching Assumptions", Int. J. Control, Vol. 45, No. 5, pp. 1527-1542, 1987.

[3] Chen, Y. H., "Adaptive Robust Control of Uncertain Systems with Measurement Noise", Automatica, Vol. 28, No. 4, pp. 715-728, 1992.

[4] Coreless, M. and G. Leitmann, "Adaptive Control of Systems Containing Uncertain Functions and Unknown Functions with Uncertain Bounds", J. Optimization Theory Applications, 41, pp. 155-168, 1983.

[5] Fu, L. C., "A New Robust MRAC Using Variable Structure Design for Relative-Degree-Two Plants", Automatica, Vol. 25, 1992.

[6] Narendra, K. S. and A. M. Annaswamy, "Stable Adaptive Systems", Prentice-Hall, 1989.

[7] Maciejowski, J. M., "Multivariable Feedback Design", Addison-Wesley, 1989.

[8] Wu, A. C., L. C. Fu, and C. F. Hsu, "On the Robust Optimal Control for Robot Manipulators", International Symposium on Implicit and Nonlinear Systems, Ft. Worth, Texas, Dec., 1992.

[9] Wu, A. C., L. C. Fu, and C. F. Hsu, "A New Decentralized Model Reference Adaptive Control for a Class of Interconnected Dynamic Systems Using Variable Structure Design", International Journal of Adaptive Control and Signal Processing, In press, 1992.

[10] Wu, A. C., L. C. Fu, and C. F. Hsu, "Robustness of Model Reference Adaptive Control Systems with Magnified Leakage Terms", EE Report, NTU, Taipei, Taiwan, R.O.C., 1992.

[11] Ioannou, P.A. and K.S. Tsakalis, "A Robust Direct Adaptive Controller", IEEE Trans. Autom. Contr., Vol. AC-31, No. 11, pp. 1033-1043, 1986.

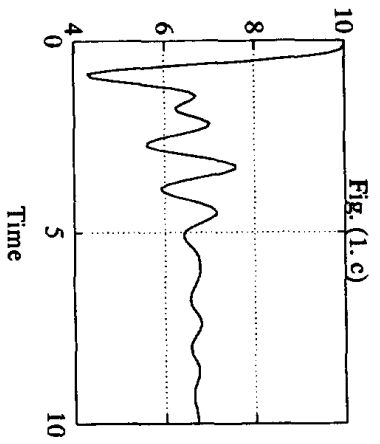

Control input u2

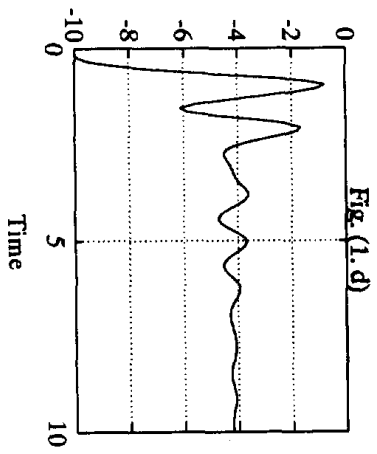

Control input $u$

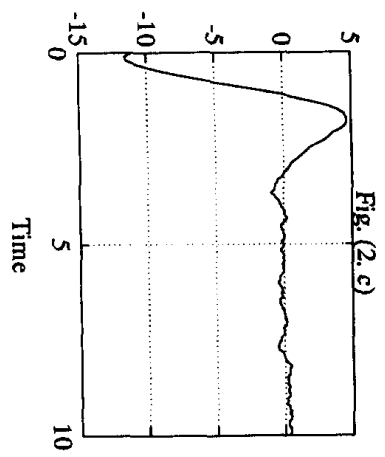

Control input $u$ in [3]

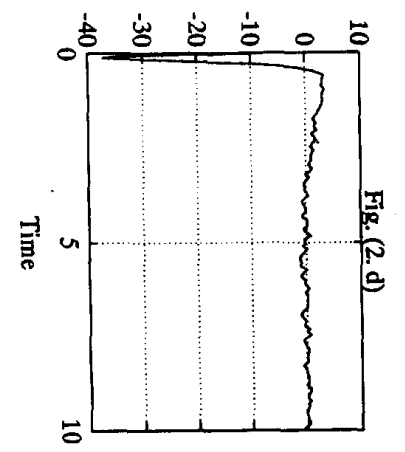

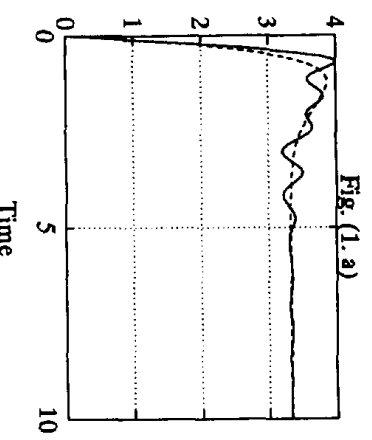

x2-solid line, xm2-dashed line

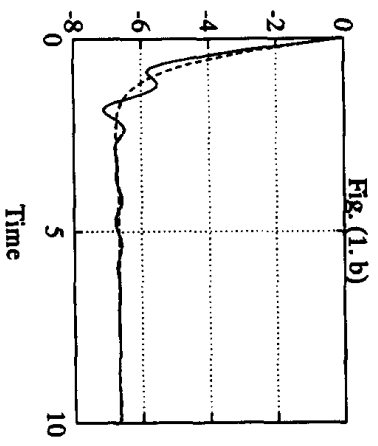

$\mathbf{x 1}$

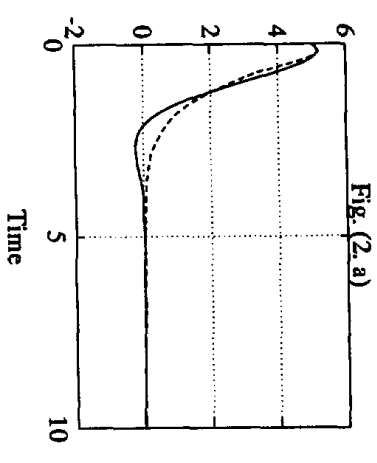

$\mathbf{x} 2$

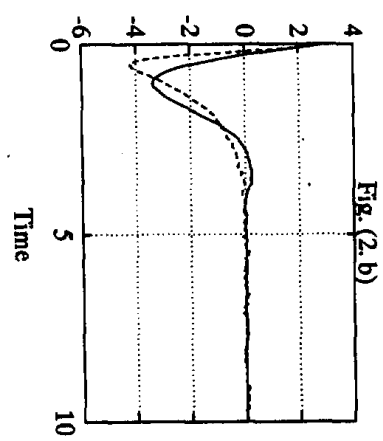

